\title{
激光冲击复合强化机理及在航空发动机部件上的 应用研究
}

\author{
李应红*，何卫锋，周留成 \\ 空军工程大学航空航天工程学院等离子动力学重点实验室, 西安 710038 \\ *E-mail: gswwd@nuaa.edu.cn
}

收稿日期: 2014-08-30; 接受日期: 2014-12-25

国家自然科学基金(批准号: 51205406, 51405507)资助项目

摘要针对激光冲击强化在航空发动机高温部件、薄叶片和叶片榫槽/榫齿等复杂部件 (位)应用的问题, 系统开展了激光冲击表面纳米化方面的研究. 本文在总结多种航空发动机 金属材料激光冲击表面纳米化表征、原理、热稳定性研究的基础上, 提出了基于表面纳米化 和残余压应力的激光冲击复合强化机理, 进而提高了激光冲击强化在高温部件上使用温度, 并介绍了薄壁结构、椎槽/榫齿等特殊部件(位)激光冲击强化工程应用的情况. 激光冲击表面 纳米化及其复合强化机理的研究工作, 拓宽了激光冲击强化的研究领域和应用范围.

关键词

激光冲击强化 航空发动机 疲劳断裂 表面纳米化 复合强化机理 高温部件

\section{1 引言}

金属构件疲劳性能与表面完整性密切相关.一 般情况下，零部件疲劳断裂特别是高周疲劳断裂往 往是在表面产生裂纹并逐渐扩展导致整体破坏. 为 提高结构可靠性, 延长使用寿命, 在不改变基体材料 性能的前提下, 表面强化技术得到了越来越多的研 究和应用.

激光冲击强化是一种高效的表面强化技术, 利 用激光冲击波的力学效应, 在金属材料表层形成大 数值残余压应力和微观组织变化, 显著提高其疲劳 强度和寿命, 是解决航空发动机高频疲劳断裂问题 的有效手段 ${ }^{[1 \sim 3]}$. 其中, 残余压应力提高金属材料的 疲劳性能机理已经有了一套比较成熟的理论, 残余 压应力主要通过降低部件承受的平均应力、降低裂纹 扩展速率甚至使裂纹闭合等方面提高材料的疲劳强
度. 美国激光冲击强化技术的发展路线也是以残余 压应力强化机制为指导, 根据部件特点, 设计激光冲 击参数, 优化残余压应力场来提高金属部件疲劳性 能 ${ }^{[4]}$.

有很多文献分析和说明了激光冲击强化的机理. 例如, Peyre 和 Fabbro ${ }^{[5]}$ 对激光冲击强化诱导残余应 力形成机制进行了描述, 并认为残余压应力是提高 疲劳性能的主要原因. Charles 等人 ${ }^{[6]}$ 对激光冲击强化 研究进行了综述, 对于提高金属材料疲劳寿命的机 理, 仍然描述为冲击后形成的残余应力改善了抗疲 劳性能. Breuer ${ }^{[7]}$, Spanrad 等人 ${ }^{[8]}$, Golden 等人 ${ }^{[9]}$, Sano 等人 ${ }^{[10,11]}$, Hatamleh ${ }^{[12]}$ 研究了激光冲击强化对金属材 料微动疲劳、外物打伤性能、焊接接头抗应力腐蚀性 能的影响，均认为激光冲击强化诱导的残余压应力 是提高疲劳性能的主要原因.

但随着激光冲击强化技术研究和应用的进一步

引用格式: 李应红, 何卫锋, 周留成. 激光冲击复合强化机理及在航空发动机部件上的应用研究. 中国科学: 技术科学, 2015, 45: 1-8 Li Y H, He W F, Zhou L C. The strengthening mechanism of laser shock processing and its application on the aero-engine components (in Chinese). Sci Sin Tech, 2015, 45: 1-8, doi: 10.1360/N092014-00234 
发展, 在一些极端服役环境和特殊部件强化中, 采用 残余压应力的强化机理至少存在三个方面的问题: 一是高温部件的强化问题. 在高温或者极端环境中, 强化后产生的残余压应力高温环境下将会大部分释 放, 强化效果显著降低 ${ }^{[13]}$; 二是榫齿/槽部位的强化 问题. 对叶片/盘的强化主要在榫齿/槽部位, 由于这 些部位形貌复杂且尺寸很小, 冲击形成的残余应力 场存在应力变化梯度, 在榫齿/槽边缘形成拉应力甚 至会引起应力集中, 影响强化效果; 三是薄叶片强化 问题. 为了提高推重比, 航空发动机叶片等结构越来 越薄, 冲击波引起的塑性变形缺乏约束, 残余压应力 难以保持易松弛, 强化效果有限. 因此, 发展激光冲 击强化新的强化机理, 具有十分迫切的工程需求, 也 是表面工程科学中的基础研究问题.

$\mathrm{Lu}$ 等人 ${ }^{[14]}$ 在纳米金属材料研究中提出表面纳米 化的概念和研究方向, 采用高能喷丸、挤压等机械方 式在金属材料表面实现了表面纳米化, 有效提高了其 力学性能. 在我们的研究中发现, 激光冲击也可实现 金属材料表面纳米化, 且形成机理和组织特征与喷丸 处理等表面机械表面纳米化方法均有所不同. 激光冲 击能量载体是冲击波, 纳米晶形成与冲击波诱导的超 高应变率塑性变形相关, 而现有文献缺乏系统研究.
为此, 开展了多种金属材料激光冲击表面纳米化的研 究, 并利用表面纳米晶和残余压应力的复合作用提高 金属材料疲劳强度. 本文以航空发动机中常用的钛合 金和高温合金为例, 介绍了激光冲击后材料的微观组 织特征、纳米化原理、热稳定性和复合强化机理，以 及激光冲击强化在航空发动机特殊部件上的典型应用 情况.

\section{2 激光冲击复合强化机理}

采用高功率脉冲激光 $\left(2 \sim 10 \mathrm{GW} / \mathrm{cm}^{2}\right)$ 对航空金属 材料作用，实现了钛合金、镍基高温合金、铝合金和 不锈钢等多种金属材料的表面纳米化 ${ }^{[15,16]}$, 如图 1 所 示. 钛合金激光冲击在表层产生的纳米晶层厚度约 1 $\mu \mathrm{m}$ ，晶粒尺寸分布为 30 300 nm; 镍基高温合金形成 的纳米晶层厚度为 $0.5 \sim 1 \mu \mathrm{m}$, 晶粒尺寸范围为 30 500 nm; 冲击 1 次表面纳米晶分布还不均匀(图 1(a) (c)), 冲击 3 5 次后，在多种金属材料表面形成 了取向随机、等轴状的纳米晶(图 1(d) (f)).

从不同金属材料激光冲击表面纳米化过程与特 征来看, 晶粒细化机制主要取决于材料本身的结构 与冲击波参数. 堆垛层错能 $(\mathrm{SFE})$ 是一个重要的参数,
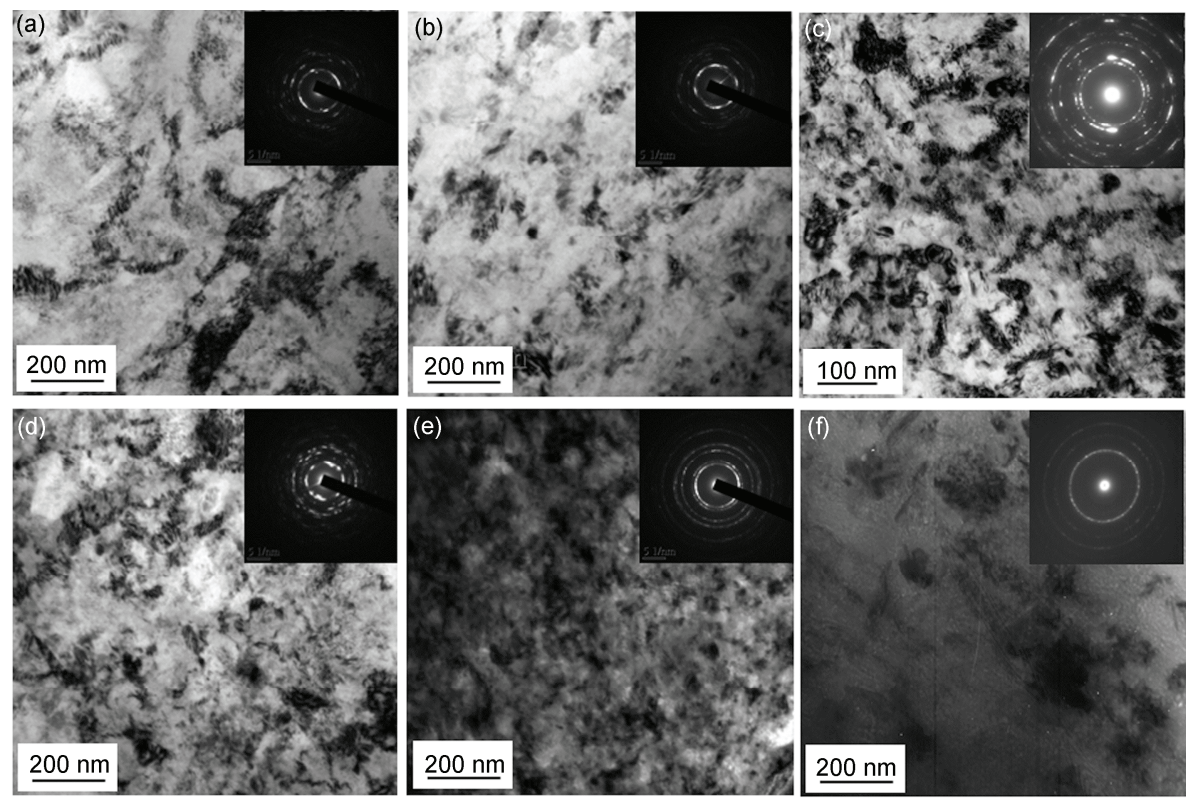

图 1 不同冲击次数后金属材料形成的表面纳米晶(功率密度 $3 \sim 9 \mathrm{GW} / \mathrm{cm}^{2}$ )

(a) TC6 钛合金; (b) TC17 钛合金; (c) K417 镍基合金; (d) TC11 钛合金; (e) 304 不锈钢; (f) 1Cr11Ni2MoV 不锈钢 
在具有高堆垛层错能材料的金属材料中, 位错运动 是冲击波作用下材料超高应变率塑性变形的主要形 式. 本文以 $\mathrm{TC} 17$ 钛合金为例, 分析高层错能材料激 光冲击表面纳米化形成机理. TC17 钛合金激光冲击 后微观组织沿深度方向特征为: 表面纳米组织、距表 面 2 5 $\mu \mathrm{m}$ 的位错胞和亚结构、 $5 \sim 20 \mu \mathrm{m}$ 的高密度位 错. 这种沿深度分布的特征也在一定程度上说明了 激光冲击波作用下, 金属材料微观组织变化规律与 演化机制.

高压等离子体冲击波是形成高应变率塑性变形 的能量载体, 是形成表面纳米化的直接动力. 当冲击 波压力达到一定阈值时, 首先在材料中形成位错, Meyers ${ }^{[17]}$ 为此提出了均匀位错成核模型, 在冲击波 作用下, 单轴应变状态产生的偏应力会使晶格扭曲, 当应力达到某一临界值, 位错在冲击波阵面上或其 附近均匀成核. 在冲击波的进一步作用下, 位错发生 滑移、积聚、相互作用、缠结、湮灭、重排等协调塑 性变形. 具有高层错能的金属及合金进行塑性变形时 会很快地形成胞状结构. 此外, 激光冲击波在金属材 料表面诱导的应变速率非常高 $\left(10^{6} \mathrm{~s}^{-1}\right)$, 可产生更高的 流变应力和更高的位错密度, 更容易形成间距在纳米 量级的位错胞, 如图 2(c) 所示.

冲击波持续作用下, 位错运动进而形成纳米晶.
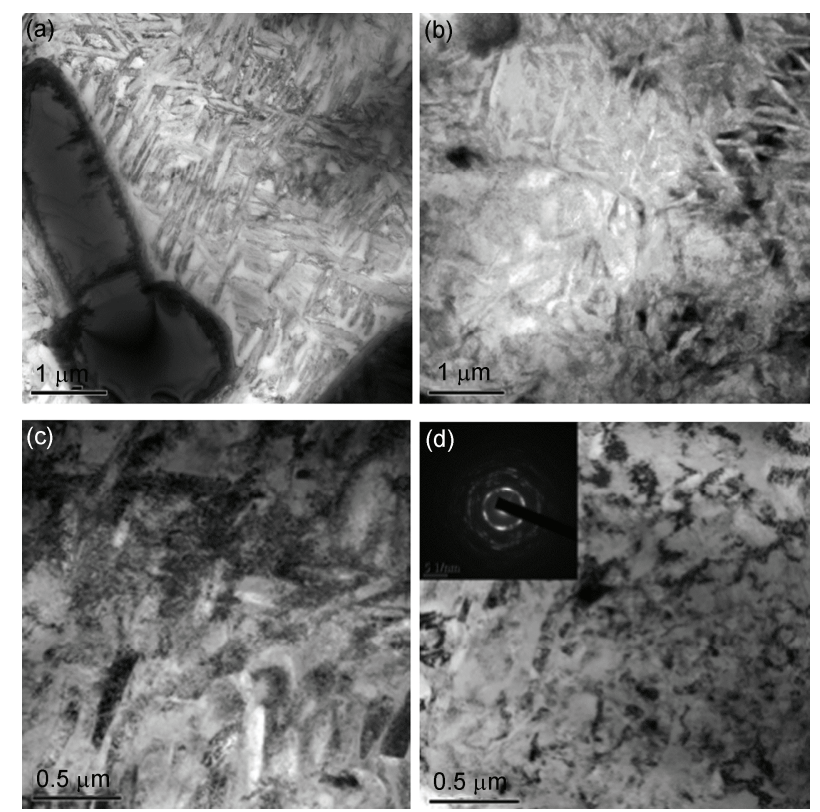

图 2 不同深度形成的典型微观组织特征

（a）基体; (b) 高密度位错; (c) 位错胞; (d) 表层纳米晶
在这个过程中, 有可能发生动态再结晶. 激光冲击形 成的塑性变形应变率非常高, 而高应变率变形过程 往往是绝热过程, 且变形做功转化为热量, 引起材料 温度升高. 在温升和剧烈塑性变形条件下, 表层材料 发生连续动态再结晶, 位错胞进一步运动诱导产生纳 米晶. 这个阶段发生两个过程: 1) 纳米尺寸的位错胞 在温升和冲击波塑性变形共同作用下, 向亚晶粒/晶粒 结构的转化; 2) 生成的亚晶界通过短程移动形成大角 度晶界, 进而形成纳米晶 ${ }^{[18 \sim 20]}$.

冲击次数对表面纳米晶尺寸和分布特征有着较 大影响. 一次冲击后, 纳米晶分布不均匀, 多次冲击 波作用后, 表面就可形成均匀的纳米晶, 增加冲击次 数可以给位错运动提供更多的时间和能量, 使得组 织变化更加均匀.

激光冲击表面纳米化后表面到深度呈梯度变化 的晶粒有效提高了金属材料的疲劳性能. 表层晶粒 尺寸细小而均匀, 在裂纹萌生阶段, 裂纹驱动力可由 更多细小的晶粒所承受, 晶内和晶界的应变梯度小, 应力集中较小, 因而材料受力均匀, 裂纹不易萌生. 在裂纹的扩展阶段, 由于纳米晶结构的晶界体积分 数高, 微裂纹将在晶界处受到阻碍, 同时一旦微裂纹 穿过晶界后, 基体晶粒存在高密度位错, 扩展方向就 会发生改变, 必然消耗更多的能量, 从而使微裂纹不 易扩展 ${ }^{[21,22]}$.

由于表面纳米晶和残余应力对疲劳性能的作用 机理是不同的, 所以在疲劳性能影响因素的分析中 往往将二者区别对待, 但是, 事实上表面纳米化过程 中必然伴随着残余应力的产生, 两者相互影响, 很难 将其对疲劳性能的影响单独分离. 在很多服役环境 下, 两者共同提高材料的疲劳强度. 激光冲击强化的 复合强化机理也是围绕这两个因素来展开研究. 对 于低温部件, 残余压应力和纳米晶同时起强化作用; 对高温部件, 残余压应力大部分松驰, 纳米晶起主要 强化作用.

\section{3 应用实例}

\section{1 薄叶片}

钛合金薄叶片是航空发动机的关键件, 使用过 程中易受到外来物打伤, 在工作载荷作用下发生疲 劳断裂. 由于叶片厚度较薄, 激光诱导的塑性变形缺 乏约束, 残余压应力在高周循环载荷作用下很快松 
弛. 此外, 由于薄叶片冲击过程中存在冲击波背面反 射、边缘效应和冲击波耦合等现象, 引起残余应力场 不均匀分布、叶边扭转宏观变形等问题.

利用小光斑/扫描冲击的方法, 在表面诱导产生 纳米晶, 提高超薄叶片的疲劳强度. 同时, 小光斑诱 导产生冲击波传播深度较浅, 形成的残余压应力层 深度也较浅, 能够防止叶片变形的发生. 还可通过特 殊透波结构, 利用阻抗匹配方法对薄壁结构内部传 播的应力波进行边界透波, 消除边界反射拉伸波, 减 弱反射波与入射波的内部耦合强度，促进表层均匀、 对称残余压应力的形成. 以 TC17 钛合金为研究对象, 设计了模拟薄叶片的振动疲劳样件, 叶片厚度为 1 $\mathrm{mm}$, 冲击区域及样品尺寸, 如图 3 所示.

采用小光斑扫描冲击的方法对其处理, 光斑直 径范围在 $0.5 \sim 2 \mathrm{~mm}$, 能量 $0.2 \sim 2 \mathrm{~J}$, 脉宽为 $5 \sim 8 \mathrm{~ns}$, 利 用升降法对振动疲劳进行考核, 如图 4 所示.

可知，激光冲击处理后，TC17 钛合金模拟薄叶 片疲劳强度提高 $20 \%$ 以上, 表面纳米晶和残余应力 共同作用是提高疲劳性能的主要原因.

\section{2 高温部件}

激光冲击形成的残余压应力在热作用下会发生 松弛, 从而削弱甚至消除强化效果. 为此, 美国激光
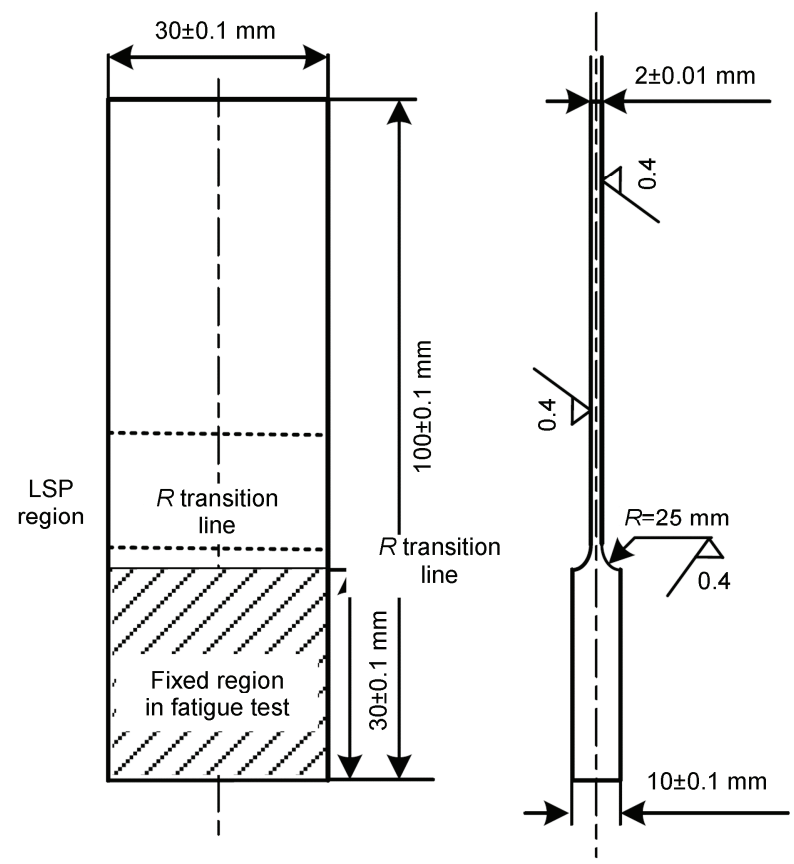

图 $3 \mathrm{TC17}$ 薄叶片样品尺寸及强化区域

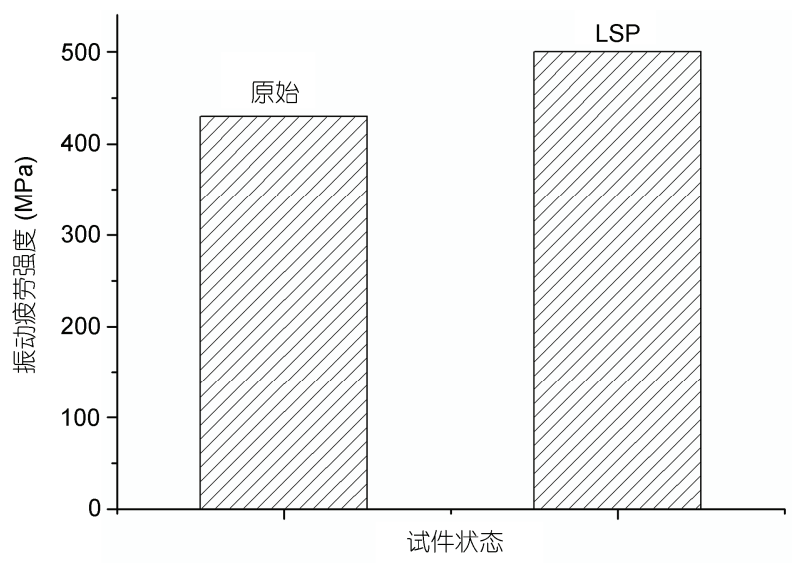

图 $4 \mathrm{TC17}$ 模拟薄叶片激光冲击振动疲劳强度对比

冲击强化规范限制了不同金属材料激光冲击后的后 续处理和使用温度范围，其中，镍基合金规定为 $538^{\circ} \mathrm{C}$ 以内. 因此, 在残余应力松弛情况下, 表面纳 米晶热稳定性是激光冲击强化在高温部件上应用的 关键问题.

K417 铸造镍基高温合金广泛用于浴轮叶片，其使 用温度在 $800^{\circ} \mathrm{C}$ 以内. 对 $\mathrm{K} 417$ 合金进行了激光冲击强 化试验, 激光脉宽 8 20 ns, 脉冲能量 2 12 J, 冲击 1 5 次, 搭接率为 $60 \%$. 分别对不同温度热处理后的冲击样 品进行残余应力和微观组织进行测试和观察. 图 5 为 K417 合金激光冲击强化后残余应力松弛情况 ${ }^{[23]}$.

可知，激光冲击诱导的残余压应力在不同温度 热处理后均有一定的松他, 且温度越高, 残余压应力 热松他程度越大. $900^{\circ} \mathrm{C} / 2 \mathrm{~h}$ 保温后, $72 \%$ 残余压应力 松弛.

图 6 为激光冲击 $\mathrm{K} 417$ 镍基合金 $900^{\circ} \mathrm{C} / 10 \mathrm{~h}$ 热处

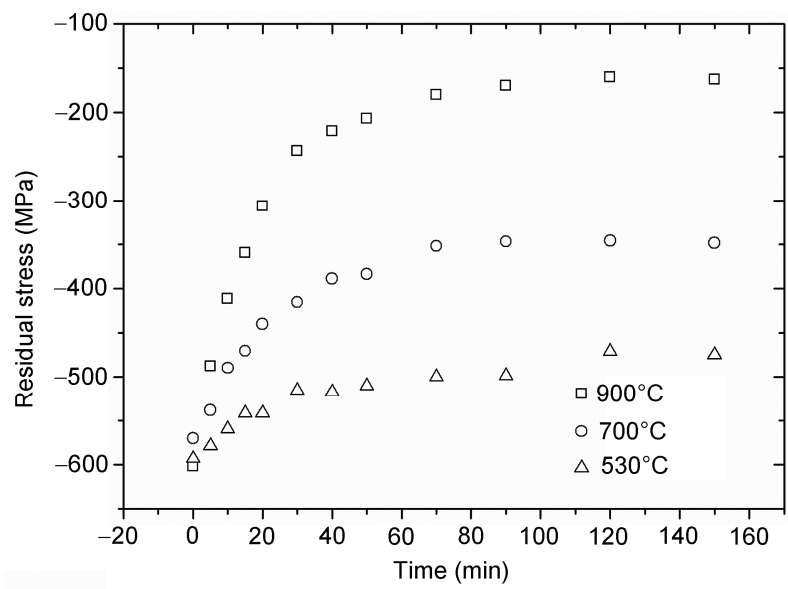

图 $5 \mathrm{~K} 417$ 镍基合金激光冲击后残余应力的热松驰 


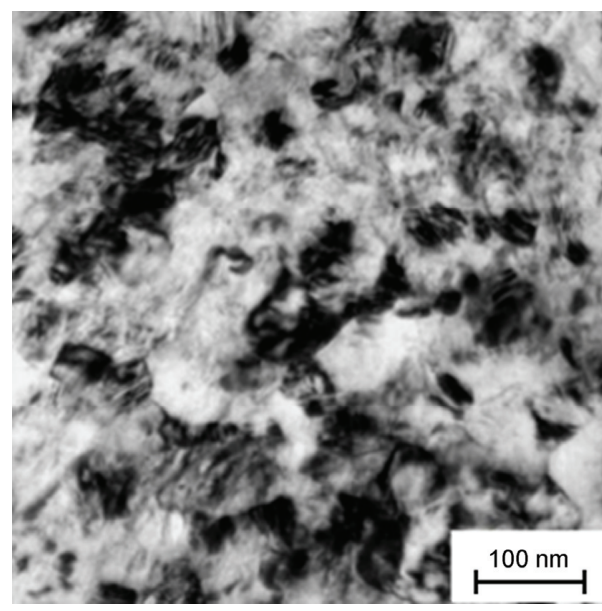

图 $6900^{\circ} \mathrm{C}$ 保温后的表面纳米晶

理后的表面微观组织. 与图 1(c)相比, 表面纳米晶没 有明显长大, 具有较好的热稳定性. 激光冲击强化低 的冷作硬化率(激光冲击强化在单次冲击小于 $1 \%$, 多 次重复冲击下只有 5\% 7\%, 而喷丸达 $40 \%$ ), 提高了 表面纳米晶的临界长大温度. 晶粒大小分布的尺寸 效应也提高了表面纳米晶的热稳定性.

为考核热处理对疲劳性能的影响, 分别对原始 样品、激光冲击强化、强化后 $900^{\circ} \mathrm{C} / 10 \mathrm{~h}$ 热处理三种 状态的试片进行振动疲劳试验对比. 图 7 和 8 分别为 标准振动疲劳样件尺寸和疲劳结果. 选用的激光冲 击参数为: 激光能量 $10.8 \mathrm{~J}$, 脉宽 $20 \mathrm{~ns}$, 光斑直径 3.4 $\mathrm{mm}$, 搭接率为 $60 \%$, 冲击 1 次.

可看到，原始 K417 试片的疲劳强度为 $110 \mathrm{MPa}$, 激光冲击强化后提高至 $285 \mathrm{MPa}, 900^{\circ} \mathrm{C} / 10 \mathrm{~h}$ 热处理 后疲劳强度为 $230 \mathrm{MPa}$, 相对未处理的试片仍提高了 1.1 倍. 热处理未明显降低激光冲击强化的效果.

\section{3 叶片榫齿/槽结构}

航空发动机浴轮叶片榫齿和轮盘榫槽部位在旋 转过程中紧密接触并随有高频振动, 其接触区域应 力水平最高, 极易产生疲劳裂纹, 如图 9 所示. 由于 榫齿/槽等部位尺寸很小(小于 $1 \mathrm{~mm}$ ), 且过渡区曲率 变化较大, 激光冲击强化中存在应力集中和应力场 分布不均匀等问题.

针对浴轮叶片榫齿/槽结构强化问题, 采用水下 小光斑/扫描冲击的方式, 多次冲击形成表层纳米组 织. 由于光斑较小, 激光冲击形成的应力场分布较为 均匀, 且冲击波传播深度较浅, 可降低应力梯度和应

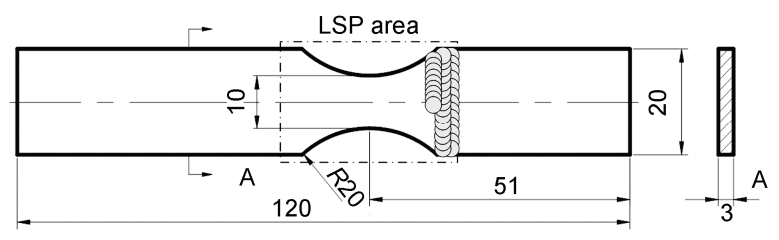

图 7 标准振动疲劳试片(单位: $\mathbf{m m}$ )

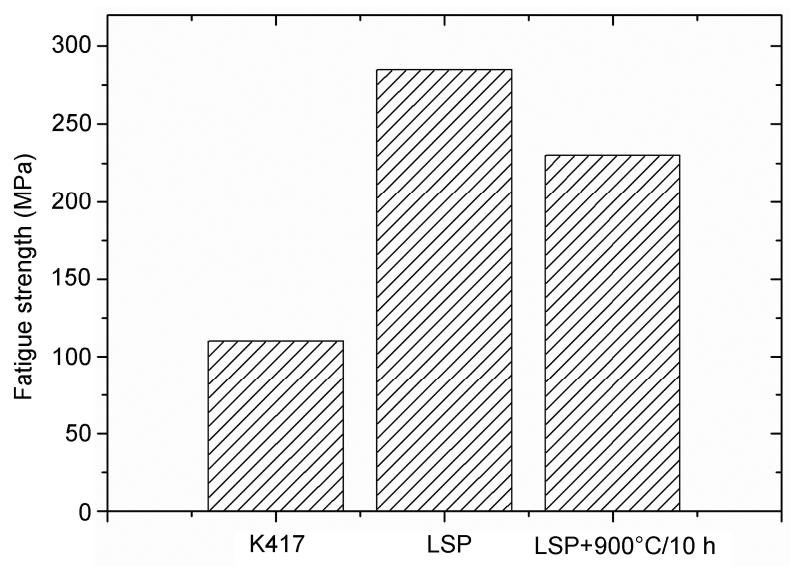

图 $8 \mathrm{~K} 417$ 试片不同处理状态的疲劳强度对比
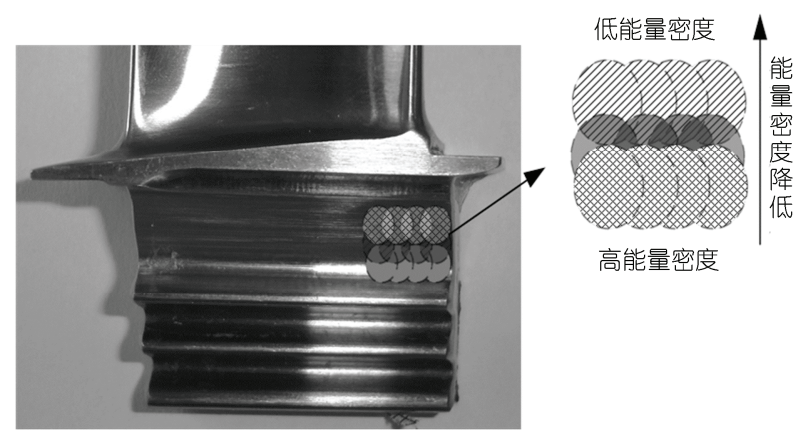

图 9 涡轮叶片榫齿/槽和不等应力冲击

力集中, 从而实现对叶片榫齿部位的激光冲击强化. 此外，还可采用“不等应力分布冲击方法”, 即利用特 殊的光斑搭接和布置方式来进行激光冲击强化, 使 应力分布合理.

采用高温高低周复合疲劳模拟航空发动机浴轮 叶片实际工作环境, 考核激光冲击强化对镍基合金浴 轮叶片榫齿/槽疲劳性能影响. 根据某型发动机典型工 作剖面, 计算出低周载荷峰值及温度载荷. 本文中选 用的振动应力为 $336 \mathrm{MPa}$, 实验温度为 $530^{\circ} \mathrm{C}$. 由于实 际航空发动机浴轮叶片振动应力小于 $336 \mathrm{MPa}$, 说明 疲劳实验结果偏安全. 图 10 为激光冲击强化前后 


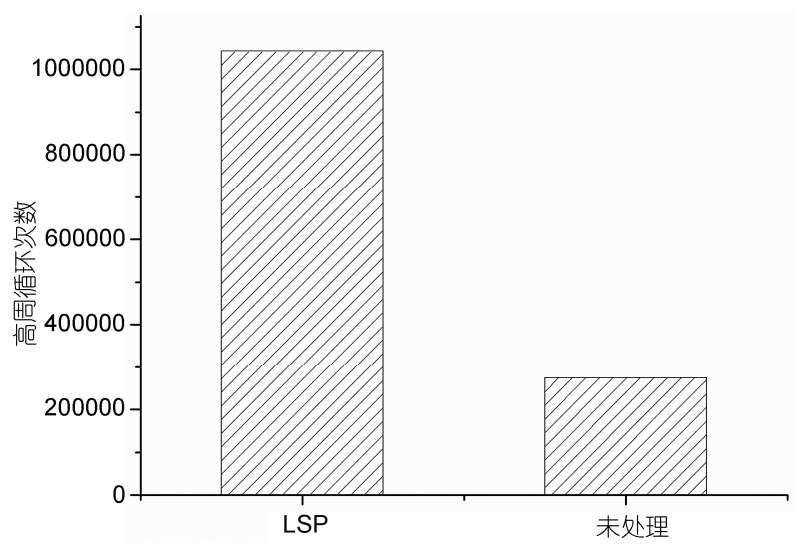

图 10 涡轮叶片激光冲击强化前后疲劳寿命对比

GH4133B 镍基高温合金浴轮叶片的中值寿命对比.

可知, 激光冲击强化后镍基合金浴轮叶片中值 寿命大幅提高, 达到未强化的 3.79 倍. 激光冲击诱导 的表面纳米晶在疲劳加载和热环境共同作用下有较 好的稳定性, 且表面纳米晶能有效提高镍基高温合 金裂纹源区抗高温氧化性能, 减缓裂纹萌生.

\section{4 小孔结构}

小孔是飞机和发动机中广泛存在的结构, 使用 过程中叠加振动机械应力和热应力等交变载荷, 存 在应力集中, 易在孔边形成疲劳裂纹.

针对小孔这种特殊结构, 采用区域性的强化方案, 沿小孔边缘区域进行蛇形三圈光斑处理, 以形成表面 纳米结构. 利用拉-拉疲劳试验, 考核激光冲击强化对 小孔试件的疲劳性能的影响, 在 $150 \mathrm{MPa}$ 的应力水平 下, 进行 $R=0.1$ 的拉-拉疲劳试验, 频率为 $25 \mathrm{~Hz}$, 强化 前后的小孔试件的疲劳寿命对比如图 12 所示.

可知, 激光冲击强化后小孔试样的疲劳寿命大 幅提高, 达到未强化的 8 倍以上.

\section{4 结束语}

我国航空工业基础薄弱, 提升航空发动机部件
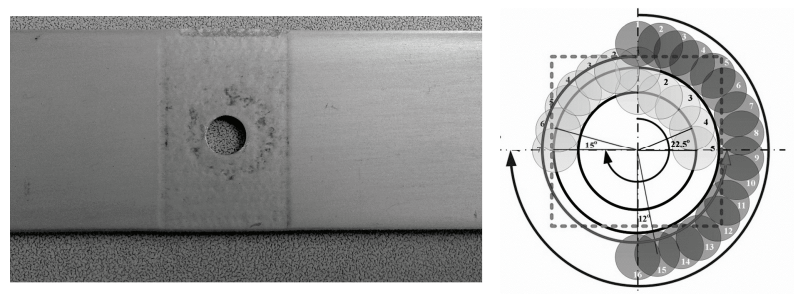

图 11 小孔强化示意图

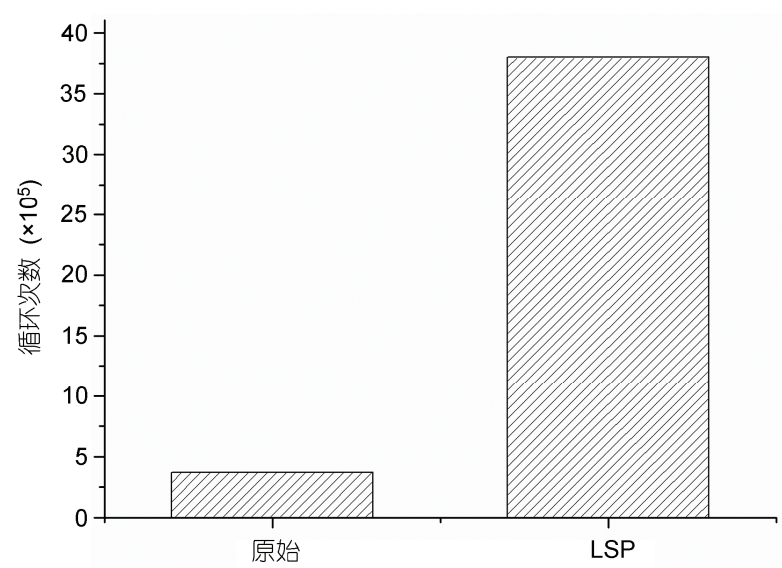

图 12 小孔强化前后疲劳寿命对比

的疲劳强度和可靠性非常迫切. 激光冲击强化新机 理的研究, 拓宽了激光冲击强化的研究领域, 解决了 现役发动机高温部件、薄壁部件等疲劳断裂等问题, 对提高航空发动机安全可靠性和提升我军装备保障 能力具有重要意义. 然而, 仍需要进行更多的科学和 技术上研究探索.

1) 冲击波作用下金属材料的超高应变率动态响 应. 激光冲击引起的材料的应变率超高 $10^{6} \mathrm{~s}^{-1}$, 当前 主要采用 $\mathrm{J}-\mathrm{C}$ 模型研究激光冲击波作用下材料的动态 响应, 该模型主要适用于 $10^{4} \mathrm{~s}^{-1}$ 应变率的动态响应研 究, 根据冲击波载荷特点构件建立超高应变率本构 模型，可更好的揭示激光冲击波作用下应力波传播 规律和部件应力应变场优化控制方法.

2) 激光冲击强化中的多尺度力学问题. 激光冲 击使表层材料微观组织结构发生变化, 从而引起宏 观力学性能改变, 目前主要是通过试验的方法研究 激光冲击强化对材料力学性能的影响, 对微观组织 与宏观性能的多尺度关系的研究函待加强.

3) 镍基高温合金单晶和定向凝固叶片强化问题. 镍基高温合金单晶和定向凝固叶片在浴轮发动机上 得到越来越广泛的应用. 单晶和定向凝固技术有效 解决了高温蠕变, 提高了浴轮叶片使用温度, 但在实 际工程应用中，单晶和定向凝固镍基合金叶片存在 齿根裂纹及断裂问题, 影响发动机安全可靠性. 由于 单晶各向异性, 在不同晶粒取向进行冲击, 其微观组 织响应方式不同, 造成力学性能的差异性较大, 选取 合适的冲击方向和冲击参数, 并建立起单晶的强化 模型，是一个具有挑战的课题.

4) 钛合金整体叶盘组合制造与修复问题. 整体 
叶盘广泛应用于先进航空发动机, 但在加工过程中, 存在少量叶片超差(尺寸不够和变形), 导致整体叶盘 的报废, 利用激光冲击成形技术实现叶片高精度局 部矫形, 提高整体叶盘的成品率. 此外, 整体叶盘在
使用过程中, 受到外来物打伤, 降低其疲劳力学性能, 利用激光冲击强化的组合修复技术, 实现损伤叶片 的高精度局部增材制造和疲劳性能提高, 是一个具 有迫切工程需求的课题.

\section{参考文献}

1 Cellard C, Retraint D, Francois M, et al. Laser shock peening of Ti-17 titanium alloy: Influence of process parameters. Mat Sci Eng A, 2012, 532: $362-372$

2 Ganesh P, Sundar R, Kumar H, et al. Studies on laser peening of spring steel for automotive applications. Opt Laser Eng, 2012, 50: 678-686

3 Thompson S R, Ruschau J J, Nicholas T. Influence of residual stresses on high cycle fatigue strength of Ti-6Al-4V subjected to foreign object damage. Int J Fatigue, 2001, 23: 405-412

4 李应红. 激光冲击强化理论与技术. 北京: 科学出版社, 2013

5 Peyre P, Fabbro R. Laser shock processing of aluminum alloys: Application to high cycle fatigue behavior. Mat Sci Eng A, 1996, 210: $102-113$

6 Montross S, Charles, Tao W, et al. Laser shock processing and its effects on microstructure and properties of metal alloys: A review. Int J Fatigue, 2002, 24: 1021-1036

7 Breuer D. Laser peening-advanced residual stress technology. Ind Heat, 2007, 74: 48-50

8 Spanrad S, Preuss M, Tong J. Cancelled laser shock peening: A solution to foreign object damage problem. The Second International Conference on Laser Peening. Francisco, 2010. 19-21

9 Golden P J, Hutson A, Sundaram V, et al. Effect of surface treatments on fretting fatigue of Ti-6Al-4V. Int J Fatigue, 2007, 29: 1302-1310

10 Sano Y, Obata M, Kubo T, et al. Retardation of crack initiation and growth in austenitic stainless steels by laser peening without protective coating. Mat Sci Eng A, 2006, 417: 334-340

11 Sano Y, Adachi Y. Enhancement of surface property by low-energy laser peening without protective coating. Key Eng Mater, 2007, 345-346: 1589-1592

12 Hatamleh O. Laser and shot peening effects on fatigue crack growth in friction stir welded 7075-T7351 aluminum alloy joints. Int J Fatigue, 2007, 29: 421-434

13 Zhou Z, Bhamare S, Ramakrishnan G, et al. Thermal relaxation of residual stress in laser shock peened Ti-6Al-4V alloy. Surf Coat Tech, 2012, 206: 4619-4627

$14 \mathrm{Lu} \mathrm{K,} \mathrm{Lu} \mathrm{J.} \mathrm{Surface} \mathrm{nanocrystallization} \mathrm{of} \mathrm{metallic} \mathrm{materials-presentation} \mathrm{of} \mathrm{the} \mathrm{concept} \mathrm{behind} \mathrm{a} \mathrm{new} \mathrm{approach.} \mathrm{J} \mathrm{Mater} \mathrm{Sci} \mathrm{Tech,} \mathrm{1999,} \mathrm{15:}$ 193-197

15 Zhou L, Li Y H, Wang C, et al. Vibration fatigue performance improvement of K417 alloy by laser shock processing and aluminizing. Rare Metal Mat Eng, 2011, 40: 1093-1096

16 Zhou L C, Li Y H, He W F, et al. Deforming TC6 Titanium alloys at ultrahigh strain rates during multiple laser shock peening. Mat Sci Eng A, 2013, 578: 181-186

17 Meyers M A. Dynamic Behavior of Materials. New York: A Wiley-interscience Publication, 1994

18 Huang C, Murthy T G, Shankar M R, et al. Temperature rise in severe plastic deformation of titanium at small strain-rates. Scripta Mater, 2008, 58: 663-666

19 Wang Y M, Bring E M, McNaney J M, et al. Deforming nanocrystalline nickel at ultrahigh strain rates. Appl Phys Lett, 2006, 88: 1-3

20 Meyers M A, Remington B A, Maddox B, et al. Laser shocking of materials: Toward the national ignition facility. Materials for Crashworthiness and Defense: Feature. JOM, 2010, 62: 24-30

21 Yang X J, Zhou J X, Ling X. Influences of surface grain size and gradient variation along depth on fatigue life of metallic materials. Mater Design, 2013, 43: 454-459

22 Yang X J, Ling X, Zhou J X. Optimization of the fatigue resistance of AISI304 stainless steel by ultrasonic impact treatment. Int J Fatigue, 2014, 61: 28-38 
23 Li Y H, Zhou L C, He W F, et al. Strengthening mechanism of a nickel-based alloy after laser shock processing at high temperature. Sci Technol Adv Mat, 2013, 14: 055010-055019

\title{
The strengthening mechanism of laser shock processing and its application on the aero-engine components
}

\author{
LI YingHong, HE WeiFeng \& ZHOU LiuCheng \\ Science and Technology on Plasma Dynamics Lab, Air Force Engineering University, Xi'an 710038, China
}

\begin{abstract}
Aiming at the application problems of laser shock processing on the aero-engine high temperature components, thin blade, blademortises/tenon teeth and other complex parts (regions), we carried out a systematical research on surface nanocrystallization induced by laser shock processing. This article summarized many studies about the characterization, theory and thermal stability of surface nanocrystallization of metal materials in various aero-engines. Moreover, we proposed a new strengthening mechanism of laser shock processing which was based on surface nanocrystallization and compressive residual stresses. Thereby, the application temperature of laser-peened high temperature components could be improved, and the engineering applications of laser shock processing on thin-wall construction, mortises/tenon teeth and other special parts (regions) were introduced. The research on laser-induced surface nanocrystallization and compound strengthening mechanism, which broadens the study area and application field of laser shock processing.
\end{abstract}

laser shock processing, aero-engine, fatigue fracture, surface nanocrystallization, compound strengthening mechanism, high temperature components

doi: 10.1360/N092014-00234 